\title{
La protéine Nef du virus HIV-1 : facteur de controverse
}

Propriétés présumées. Le virus de l'immunodéficience humaine (HIV) présente une organisation génomique complexe, avec l'identification à ce jour de dix gènes codant pour des protéines structurales et régulatrices de l'expression génique virale. Parmi les protéines de régulation, $\mathrm{Nef}$ ou negative-regulatory factor (précédemment nommée 3'orf, orf-B, E' ou $F$ ) ne semble pas indispensable à la réplication et aux effets cytopathogènes du HIV [1, 2]. La protéine était jusqu'alors présumée exercer une inhibition de la transcription de l'ensemble des gènes viraux, participant à l'établissement et à la maintenance de la phase latente du HIV au sein des cellules infectées [2-5]. En effet, il a été rapporté une forte augmentation de la cytopathogénicité et du taux de réplication de souches mutantes du HIV déficientes pour le gène nef [3]. Parallèlement, certaines propriétés ou caractéristiques particulières de la protéine Nef ont été définies [6] : (a) l'induction d'une diminution de l'expression du récepteur CD4 (cible du HIV) à la surface des lymphocytes infectés par le virus de la vaccine exprimant le gène nef; (b) des propriétés biochimiques comparables à celles des protéines $\mathrm{G}$ cellulaires : capacité de fixation du guanosine 5'-triphosphate (GTP) associée à l'activité GTPase ; (c) une activité d'autophosphorylation ; (d) la phosphorylation potentielle (thréonine en position 15) de certains variants de Nef (HIV-1) par la protéine kinase C ainsi qu'une homologie de ses séquences de phosphorylation avec celles des proto-oncogènes src et ras, et (e) la présence éventuelle d'un adhésiotope (séquence tétrapeptidique RFDS de la protéine Nef du HIV-1) sion cellulaire, au récepteur CD4 des lymphocytes (séquence RADS), ou aux chaînes $\beta$ des antigènes de classe II du complexe majeur d'histocompatibilité. Les travaux réalisés par la société Transgène (Strasbourg) et l'Institut Pasteur (Paris) ont mis en évidence l'apparition d'une souche virale particulièrement cytopathogène à l'issue d'étapes successives de culture du HIV-1 (isolat bru) sur des cellules lymphocytaires humaines de lignée CEM. L'analyse biochimique de ce nouvel isolat a révélé la présence de plusieurs mutations au sein de la protéine Nef, notamment la substitution des résidus de thréonine 15 en alanine et d'acide aspartique 186 en asparagine. Les mutations de la protéine affectent principalement la capacité de phosphorylation par la protéine kinase $\mathrm{C}$, la fixation du GTP et la régulation de l'expression cellulaire du récepteur CD4. Spire et al. ont également décrit l'existence d'une souche du HIV-1 très cytopathogène, nommée NDK, dont le produit du gène nef présente des mutations localisées au niveau de sites identiques [7]. Ces données suggèrent qu'au cours de l'infection virale, les mutations de la protéine Nef pourraient potentiellement répondre de la transition du HIV de l'état de latence, qui constitue un processus d'échappement du virus aux défenses immunitaires de l'hôte, à l'état de forte virulence. Cette transition devrait se traduire par l'évolution clinique du sujet infecté vers un SIDA. Établir la validité de cette hypothèse nécessite cependant la détermination structurale de la protéine après isolement du HIV chez l'homme à divers stades de l'infection virale. Malgré ces résultats concordants sur la fonction biologique attribuée à la protéine Nef, le rôle apparent de régulateur négatif a été controversé par plusieurs travaux récents pour lesquels il n'a été observé aucun effet significatif de la molécule sur l'expression du HIV [8-10]. En effet, Kim et al. ont comparé la capacité de réplication d'un isolat du HIV-1 déficient pour le gène nef (nef-) à celle de l'isolat sauvage exprimant ce gène (nef ${ }^{+}$). Les résultats montrent que la réplication virale dans les différents types cellulaires testés est indépendante de la présence du gène nef. En accord avec ces travaux, Bachelerie et al. ont démontré que l'expression de la protéine Nef du HIV-1 dans une lignée astrocytaire humaine n'affecte pas la transcription des gènes sous le contrôle du LTR (long terminal repeat) du HIV [11]. Par conséquent, les données actuelles concernant la protéine Nef sont contradictoires et soulignent la nécessité de réévaluer la fonction biologique de la molécule. Parallèlement, l'activité GTPase de la protéine a été remise en question par Kaminchi et al. selon lesquels l'activité enzymatique préalablement décrite pourrait être associée à la présence de protéines bactériennes contaminant la préparation de la protéine Nef produite par génie génétique [12].

Caractéristiques structurales. Le gène nef correspond à un cadre de lecture polymorphe localisé au niveau du LTR à l'extrémité 3' du génome viral [1]. Absent des génomes rétroviraux " classiques ", ce cadre de lecture est conservé dans la majorité des isolats du HIV-1, HIV-2 et du virus de l'immunodéficience simienne de type 1 (SIV-1), ce qui suggère toutefois l'importance fonctionnelle de la protéine Nef dans le cycle de réplication virale ou les effets biologiques 
produits par le virus au sein des cellules infectées. Le produit du gène nef (la protéine Nef) est issu de la traduction d'un ARN messager ayant subi l'excision de deux introns par rapport au transcrit viral primitif ou ARNm génomique. Il s'agit d'une molécule de 206 et 255 résidus d'acides aminés pour le HIV-1 (isolat bru) et le HIV-2 (isolat rod), respectivement. La protéine présente une variabilité structurale inter-isolats inférieure à $17 \%$ et apparaît parti- culièrement polymorphe au niveau des régions $\mathrm{N}$ - et $\mathrm{C}$-terminales (20 à $40 \%$ ). La molécule ne semble pas glycosylée, bien qu'il existe deux sites potentiels de $\mathrm{N}$-glycosylation, tandis que l'extrémité $\mathrm{N}$-terminale est myristilée suggérant une interaction possible de la protéine avec la face interne de la membrane cellulaire à partir de laquelle elle est présumée exercer son activité biologique. La modification post-traductionnelle de la protéine par myristilation paraît essentielle à son activité modulatrice de l'expression du récepteur CD4 lymphocytaire.

Mode d'action possible. Le site d'action putatif de la protéine est la séquence NRE (negative-regulatory element) localisée à l'extrémité 5' du LTR de l'ADN proviral, entre les nucléotides 340 et 185 en amont du site d'initiation de la synthèse d'ARN. Cette séquence est intrinsèquement inhibitrice de la transcription par son action en cis sur le site

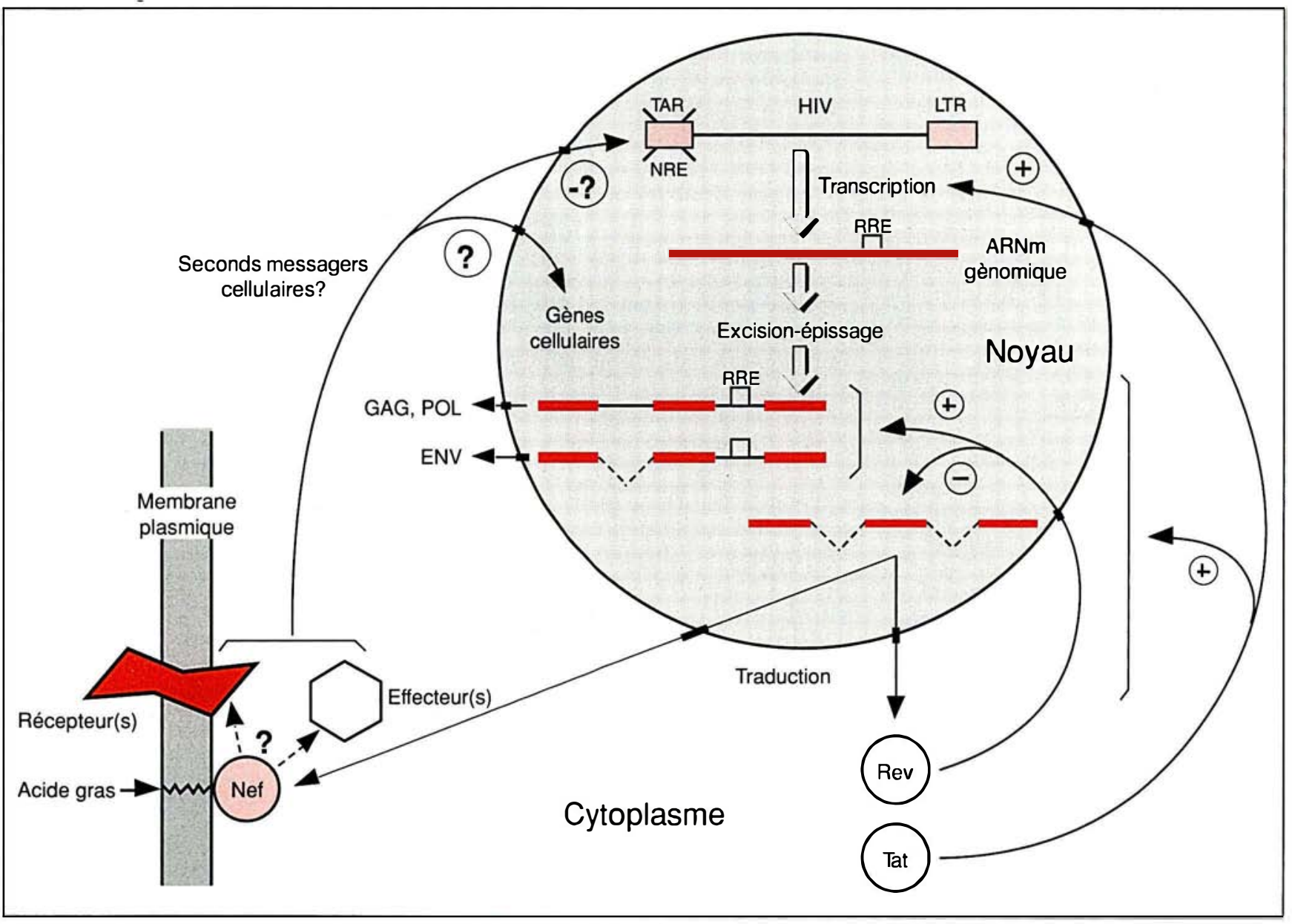

Figure 1. Mécanismes de la régulation génétique du HIV. Le génome viral intégré (provirus) est encadré par deux longues répétitions terminales (LTR) incluant les éléments génétiques nécessaires à la transcription ainsi que les séquences régulatrices NRE (negative-regulatory element) et TAR (Tat-responsive element). L'ARN messager (ARNm) génomique, produit de la transcription de l'ADN proviral, subit un mécanisme d'excision-épissage engendrant divers types d'ARNm traduits dans le cytoplasme en protéines structurales (Gag, Pol, Env) et régulatrices (Tat, Rev, Nef...). La protéine Tat trans-active l'expression du provirus par son interaction avec la séquence TAR du LTR. La régulation est exercée au niveau transcriptionnel, sur l'initiation et l'anti-terminaison précoce de la transcription, et posttranscriptionnel, sur la stabilité et l'efficacité traductionnelle des ARNm. La protéine Rev interagit avec la séquence RRE (Rev-responsive element) et conduit à stabiliser sélectivement les ARNm codant pour les protéines structurales. La protéine Nef, ancrée par un acide gras (acide myristilique) à la face interne de la membrane plasmique, pourrait agir sur la séquence NRE du LTR par l'intermédiaire de facteurs cellulaires spécifiques. Cette interaction hypothétique conduirait à la répression de la transcription de l'ensemble des gènes viraux.

$m / s n^{\circ} 1$, vol. 7 , jancier 91 
promoteur [4]. La protéine Nef, préférentiellement localisée dans le cytoplasme, agirait à distance sur la séquence NRE par l'intermédiaire de facteurs cellulaires activés (peut-être par phosphorylation) participant à la transmission de son message chimique au sein du noyau (figure 1). Un modèle a été proposé par Guy et al. sur un mécanisme possible de régulation par la protéine Nef des facteurs cellulaires se liant à la séquence NRE [13]. Dans ce modèle, un répresseur associé à la séquence NRE serait responsable d'une inhibition de la transcription au sein de la cellule " au repos " et serait normalement déplacé, après stimulation cellulaire, par des facteurs activateurs favorisant la transcription; la protéine Nef pourrait bloquer la fixation des activateurs sur la séquence NRE conduisant à maintenir l'inhibition de la transcription. Par ce mécanisme, la protéine se comporterait comme un anti-activateur de l'expression du HIV et interférerait avec la transcription de gènes cellulaires spécifiques (ex. : CD4). La protéine Nef devrait parallèlement agir en interaction coopérative avec les autres facteurs de régulation du HIV (notamment Tat et $\mathrm{Rev}$ ), et de manière étroitement dépendante des mécanismes physiologiques de la cellule infectée.

Réponse immunitaire anti-Nef et diagnostic précoce de l'infection par le HIV. L'antigénicité de la protéine Nef chez l'homme infecté par le HIV a été confirmée par différentes techniques immunologiques. Ces études ont révélé l'existence d'anticorps anti-Nef chez 30 à $85 \%$ des patients séropositifs pour le HIV-1 $[1,14,15]$. Par ailleurs, il semble n'exister aucune corrélation entre la présence de ces anticorps et le stade clinique des sujets infectés. Généralement, le diagnostic de l'infection par le HIV repose sur la détection sérologique des anticorps dirigés contre les protéines structurales (Gag, Pol, Env), marqueurs de la séroconversion. Des études basées sur l'isolement du HIV par culture cellulaire ou sur la détection de l'ADN proviral des lymphocytes par la technique d'amplification génomique (polymerase chain reaction) ont permis d'identifier des patients séronégatifs infectés par le HIV. Cette période immunosilencieuse entre l'infection virale et la séroconversion est de durée très variable et peut s'étendre suivant les cas de quelques semaines jusqu'à plus de 35 mois. Les travaux de Ranki et al., Ameisen et al.; et Gombert $e t$ al., suggèrent l'apparition précoce des anticorps anti-Nef avant la phase de séroconversion des sujets infectés par le HIV [16-19]. Ces résultats sont en désaccord avec les travaux de Reiss et al., Gluckman et al., et Bahraoui et al., pour lesquels les anticorps anti-Nef sont détectés simultanément aux anticorps dirigés contre les protéines structurales, infirmant la validité des anticorps antiNef comme marqueurs sérologiques précoces de l'infection virale $[15,20$, 21]. Selon Ranki et al., il semble que la détection des anticorps anti-Nef ne soit pas systématiquement associée à une infection par le HIV et pourrait résulter de l'existence d'une immunoréactivité croisée avec une protéine non identifiée d'origine cellulaire ou virale [22]. Les études de l'antigénicité de la protéine Nef du HIV-1 à l'aide de peptides synthétiques [19, $23,24]$ ont également révélé l'immunodominance des extrémités $\mathrm{N}$ - et $\mathrm{C}$ terminales et permis de localiser des déterminants antigéniques au niveau des régions suivantes : 8-16, 52-60, 80-90, 98-112, 115-127, 136-147, 148-161, 158-169, 180-190, 197-206 (épitopes B) et 73-82, 113-128, 186-206 (épitopes T). Malgré la variabilité de la protéine Nef, l'analyse structurale de la molécule montre une homologie de position des épitopes B entre les différents isolats du HIV-1. Il est intéressant de remarquer que la région antigénique 180-190 inclue l'adhésiotope RFDS (séquence 184-187). Ainsi, les anticorps anti-Nef dirigés contre cette région pourraient se comporter comme des anti-HLA de classe II et participer au déficit immunitaire en bloquant certaines interactions moléculaires nécessaires au bon fonctionnement du système immunitaire [25]. La définition des régions potentiellement antigéniques de la protéine constitue une étape essentielle pour la compréhension des mécanismes de la réponse immunitaire de l'hôte et devrait faciliter la mise au point de compositions vaccinales anti-HIV.

La protéine Nef : régulateur négatif de la réplication du HIV ? Par une régulation négative exercée sur la réplication virale, la protéine Nef pourrait conduire à développer de nouvelles stratégies thérapeutiques en étant une cible potentielle pour le contrôle du cycle réplicatif du HIV. Cependant, l'ensemble des travaux publiés montre que le mode d'action de la protéine et son implication précise dans la physiopathologie de l'infection par le HIV sont incertains et restent à clarifier. La molécule exerce-t-elle réellement une régulation négative sur la réplication du HIV et, dans l'affirmative, quel est le mécanisme moléculaire mis en jeu : inhibition de la transcription ou modulation post-transcriptionnelle ? Présente-t-elle une activité enzymatique protéine $\mathrm{G}$ like ? Les anticorps anti-Nef sont-ils détectables avant la phase de séroconversion des patients contaminés? La précocité de la réponse immunitaire anti-Nef lors de l'infection est-elle à l'origine de la mise en latence du HIV par la destruction des cellules infectées en cours d'assemblage de particules virales? La réponse à de nombreuses questions non résolues devrait favoriser la compréhension du cycle infectieux du HIV, ce qui nécessite au préalable une réévaluation précise de la fonction biologique, des propriétés biochimiques et antigéniques de la protéine : "Nef is Nef?"

Jean-Marc Sabatier, Jurphaas Van Rietschoten, Claude Granier, Elmostafa Bahraoui

Cnrs UA 1179, laboratoire de biochimie, Faculté de Médecine Nord, bd PierreDramard, 13326 Marseille Cedex 15, France. 
1. Allan JS, Coligan JE, Lee TH, et al. A new HTLV-III/LAV encoded antigen detected by antibodies from AIDS patients. Science $1985 ; 230$ : 810-3.

2. Terwilliger E, Sodroski JG, Rosen CA, Haseltine WA. Effects of mutations within the 3' orf open reading frame region of human T-cell lymphotropic virus type III (HTLV-III/LAV) on replication and cytopathogenicity. J Virol 1986; 60 : 754-60. 3. Luciw PA, Cheng-Mayer C, Levy JA. Mutational analysis of the human immunodeficiency virus: the orf-B region downregulates virus replication. Proc Natl Acad Sa USA 1987 ; 84 : 1434-8.

4. Ahmad N, Venkatesan S. Nef-protein of HIV-1 is a transcriptional repressor of HIV-1 LTR. Science $1988 ; 241$ : 1481-5. 5. Niederman TM, Thielan BJ, Ratner L. Human immunodeficiency virus type 1 negative factor is a transcriptional silencer. Proc Natl Acad Sci USA 1989 ; 86 : 1128-32. 6. Guy B, Kieny MP, Riviere $\mathrm{Y}$, et al. HIV F/3' orf encodes a phosphorylated GTP-binding protein resembling an oncogene product. Nature 1987 ; 330 : 266-9. 7. Spire B, Sire J, Zachar V, et al. Nucleotide sequence of HIV-1 NDK : a highly cytopathic strain of the human immunodeficiency virus. Gene $1989 ; 81$ : 275-84.

8. Kim S, Ikeuchi K, Byrn R, Groopman J, Baltimore D. Lack of a negative influence on viral growth by the nef gene of human immunodeficiency virus type 1 . Proc Natl Acad Sci USA 1989 ; 86 : 9544-8. 9. Hammes SR, Dixon EP, Malim MH, Cullen BR, Greene WC. Nef protein of human immunodeficiency virus type 1 : evidence against its role as a transcriptional inhibitor. Proc Natl Acad Sci USA 1989; 86 : 9549-53.

10. Cheng-Mayer C, Iannello $P$, Shaw $K$, Luciw PA, Levy JA. Differential effects of nef on HIV replication: implications for viral pathogenesis in the host. Science 1989 ; 246: 1629-32.

11. Bachelerie F, Alcami J, Hazan U, et al. Constitutive expression of human immunodeficiency virus (HIV) Nef-protein in human astrocytes does not influence basal or induced HIV long terminal repeat activity. J Virol $1990 ; 64: 3059-62$.

12. Kaminchi J, Bashan N, Pinchasi D, et al. Expression and biochemical characterization of human immunodeficiency virus type 1 nef gene product. J Virol $1990 ; 64$ : 3447-54.

13. Guy B, Acres B, Kieny MP, Lecocq JP. DNA binding factors that bind to the negative regulatory element of the human immunodeficiency virus-1 : regulation by

$\mathrm{m} / \mathrm{s} \mathrm{n}^{\circ} 1$, vol. 7 , janvier 91
Nef. J Acq Immun Def Synd 1990; 3 : 797-09.

14. Sabatier JM, Clerget-Raslain B, Fon$\tan G$, et al. Use of synthetic peptides for the detection of antibodies against the Nef regulating protein in sera of $\mathrm{HIV}$-infected patients. AIDS $1989 ; 3: 215-20$.

15. Reiss P, De Ronde A, Lange JMA, et al. Antibody response to the viral negative factor (Nef) in HIV-1 infection : a correlate of levels of HIV-1 expression. AIDS $1989 ; 3: 227-33$

16. Ranki A, Valle SL, Krohn M, et al. Long latency precedes overt seroconversion in sexually transmitted human immunodeficiency virus infection. Lancet 1987 ; ii : 589-93

17. Ameisen JC, Guy B, Chamaret S, et al. Antibodies to the Nef protein and to $\mathrm{Nef}$ peptides in HIV-1-infected seronegative individuals. AIDS Res Hum Retrov 1989 ; 5 : 279-91.

18. Ameisen JC, Guy B, Chamaret S, et al. Persistent antibody response to HIV - 1 negative regulatory factor in HIV-1 infected seronegative persons. $N$ Engl J Med $1989 ; 320: 251-2$.

19. Gombert FO, Blecha W, Tahtinen $M$ et al. Antigenic epitopes of Nef-proteins from different HIV strains as recognized by sera from patients with manifest and latent HIV infection. Virology $1990 ; 176$ : 458-66. 20. Gluckman JC, Fretz-Foucault C, Rouzioux $\mathrm{C}$, et al. Lack of anti-p27 Nef antibody detection in HIV seronegative highrisk individuals. AIDS $1989 ; 3$ : 855-6. 21. Bahraoui E, Ben jouad A, Sabatier JM, et al. Relevance of anti-Nef antibody detection as an early serologic marker of HIV infection. Blood $1990 ; 76: 1-8$.

22. Ranki A, Jarvinen $K$, Valle SL, Nurmibaakso P, Krohn K. Antibodies to recombinant HIV-1 Nef-protein detected in HIV-1 infection as well as in nonrisk individuals. J Acq Immun Def Synd 1990; 3 : 348-55.

23. Sabatier JM, Fontan G, Loret E, et al. Large fragments of Nef-protein and gp 110 envelope glycoprotein from HIV-1 : synthesis, $\mathrm{CD}$ analysis and immunoreactivity. Int J Peptide Protein Res 1990 ; 35 : 63-72.

24. Bahraoui E, Yagello M, Billaud JN, et al. Immunogenicity of the human immunodeficiency virus (HIV) recombinant nef gene product. Mapping of T-cell and B-cell epitopes in immunized chimpanzees. AIDS Res Hum Retrov 1990 ; 6 : 1087-98.

25. Auffray C. Un modèle moléculaire de l'interaction entre l'antigène $T 4$ et les antigènes HLA de classe II ou le virus LAV. CR Acad Sci 1986 ; 302 : 287-92. 